K.A. van der Hucht, G. Koenigsberger \& P.R.J. Eenens, eds.

\title{
Evolution and nucleosynthesis of Wolf-Rayet stars
}

\author{
Knut Jørgen Røed Ødegaard \\ Institute of Theoretical Astrophysics, University of Oslo, \\ PO Box 1029, Blindern, N-0315 Oslo, Norway
}

\begin{abstract}
Using a dynamical stellar code, detailed evolutionary models have been computed that follow the evolution and nucleosynthesis of massive stars from the pre-MS up through their most advanced stages.
\end{abstract}

\section{Introduction}

The present calculations were done with an extended version of the Göttingen dynamical stellar evolution code. This code includes a large and flexible nuclear network consisting of 174 nuclear species from $n$ and ${ }^{1} \mathrm{H}$ to ${ }^{71} \mathrm{Ge}$. The nuclear species are linked by up to 1742 nuclear reactions, including $s$-process reactions and reactions able to cover all evolutionary stages up through Si-burning. Both semi-convection and overshooting $\left(0.2 \mathrm{H}_{\mathrm{p}}\right)$ are included. The ingredients of the models and results for other evolutionary stages are presented in $\varnothing$ degaard (1996) and Ødegaard (1997). The mass loss rates for the WR stages are computed according to the prescription of Langer (1989).

\section{The models}

The model sequence for a star with solar metallicity and initial (maximum) mass $120 \mathrm{M}_{\odot}$ will be discussed. Due to extreme mass loss during the LBV and WR stages, the mass is reduced to $4.76 \mathrm{M}_{\odot}$ at the pre-SN stage. In these models convective zones are not assumed to be chemically homogenous. The mixing due to convection, semi-convection and overshooting is followed in great detail. During late stages, $\mu$-gradients often occur inside convective zones because (a) material mixed in from top and bottom has not had time to fully mix; (b) time scales of nucleosynthesis and mixing may become comparable; and (c) extention of convective zone may change. Standard mixing-length theory had to be modified to account for these phenomena. During late evolutionary stages, burning shells cause a complex internal structure with several interacting convective and semi-convective zones.

During transition from core Ne- to core O-burning, the center is partially degenerate $(\eta=5.315)$ and has $T_{\mathrm{c}}=1.84 \times 10^{9} \mathrm{~K}$ and $\rho=3.38 \times 10^{7} \mathrm{~g} \mathrm{~cm}^{-3}$. The interior is extremely complex at this stage. Several very active burning shells are present. Numerous, but very thin, convective layers cause mixing. In some of these, the local Eddington luminosity is exceeded by large factors. Probably due to the degeneracy, some burning shells are flash-like. The shells are interacting in an extremely complex and dynamical manner. Before onset of core O-burning, 
the temperature maximum is off-center and the nuclear energy-production rate is negative out to the very sharp C-burning shell.

\section{Nucleosynthesis}

Due to the detailed description of mixing, nuclear reactions and large nuclear network, internal abundances as well as surface abundances and effects of the wind on the surrounding space can be calculated. The surface abundances depend on the uncertain mass loss rates of WR stars. With a $3 \%$ higher $\dot{M}$ the abundance of ${ }^{17} \mathrm{O}$ would be 30 times higher $\left(4 \times 10^{-8}\right)$ and the ${ }^{4} \mathrm{He}$ abundance 0.12 . Is the $\dot{M}(\mathrm{WR})$ reduced by one half, ${ }^{4} \mathrm{He}$ would increase to 0.35 or more.

Table 1. Surface abundances at the WC stage (mass fractions), accumulated mass loss and mean mass fraction $X_{\dot{M}}$ of this mass loss (last column) for some nuclear species.

\begin{tabular}{l|c|c|c|c|c}
\hline element & init. abund. & WC abund. & prod. & acc. $\dot{M} / \mathrm{M}_{\odot}$ & mean $X_{\dot{M}}$ \\
& & & & & \\
\hline${ }^{4} \mathrm{He}$ & 0.26 & 0.217 & 0.83 & 70.4 & 0.61 \\
${ }^{12} \mathrm{C}$ & $3.2 \times 10^{-3}$ & 0.499 & 156 & 10.8 & 0.094 \\
${ }^{16} \mathrm{O}$ & 0.01 & 0.216 & 21.6 & 1.58 & 0.014 \\
${ }^{20} \mathrm{Ne}$ & $1.7 \times 10^{-3}$ & $8.8 \times 10^{-4}$ & 0.52 & 0.157 & $1.4 \times 10^{-3}$ \\
${ }^{21} \mathrm{Ne}$ & $4.35 \times 10^{-6}$ & $2.56 \times 10^{-2}$ & 5890 & 1.27 & 0.011 \\
${ }^{22} \mathrm{Ne}$ & $1.37 \times 10^{-4}$ & $3.20 \times 10^{-2}$ & 234 & 1.53 & 0.013 \\
${ }^{23} \mathrm{Na}$ & $3.52 \times 10^{-5}$ & $1.69 \times 10^{-4}$ & 4.80 & 0.0163 & $1.41 \times 10^{-4}$ \\
${ }^{24} \mathrm{Mg}$ & $5.42 \times 10^{-4}$ & $2.89 \times 10^{-3}$ & 5.33 & 0.0901 & $7.82 \times 10^{-4}$ \\
${ }^{28} \mathrm{Si}$ & $6.88 \times 10^{-4}$ & $2.24 \times 10^{-4}$ & 0.33 & 0.0580 & $5.03 \times 10^{-4}$ \\
${ }^{60} \mathrm{Ni}$ & $2.06 \times 10^{-5}$ & $2.80 \times 10^{-5}$ & 1.36 & $2.82 \times 10^{-3}$ & $2.45 \times 10^{-5}$ \\
${ }^{62} \mathrm{Ni}$ & $2.92 \times 10^{-6}$ & $3.52 \times 10^{-5}$ & 12.1 & $1.96 \times 10^{-3}$ & $1.70 \times 10^{-5}$ \\
${ }^{63} \mathrm{Cu}$ & $6.06 \times 10^{-7}$ & $1.51 \times 10^{-5}$ & 24.9 & $8.06 \times 10^{-4}$ & $6.99 \times 10^{-6}$ \\
${ }^{71} \mathrm{Ga}$ & $2.9 \times 10^{-8}$ & $9.37 \times 10^{-4}$ & 32300 & $4.17 \times 10^{-2}$ & $3.6 \times 10^{-4}$ \\
${ }^{70} \mathrm{Ge}$ & $4.6 \times 10^{-8}$ & $4.83 \times 10^{-5}$ & 1050 & $2.27 \times 10^{-3}$ & $2.0 \times 10^{-5}$ \\
& & & & & \\
\hline
\end{tabular}

Acknowledgments. This work has received support from The Research Council of Norway (Programme for Supercomputing) through a grant of computing time.

\section{References}

Langer, N. 1989, A\&A 220, 135

Ødegaard, K.J.R. 1996, in: J.-M. Vreux, A. Detal, D. Fraipont-Caro, E. Gosset \& G. Rauw (eds.), Wolf-Rayet Stars in the Framework of Stellar Evolution, Proc. $33^{\text {rd }}$ Liège Int. Astroph. Coll. (Liège: Univ. of Liège), p. 81

Ødegaard, K.J.R. 1997, in: A. Nota \& H. Lamers (eds.), Luminous Blue Variables: Massive Stars in Transition, ASP-CS 120, 154 